\title{
Hypocalcemia, CTCAE
}

National Cancer Institute

\section{Source}

National Cancer Institute. Hypocalcemia, CT CAE. NCI Thesaurus. Code C143565.

A disorder characterized by laboratory test results that indicate a low concentration of calcium (corrected for albumin) in the blood. 